\title{
Peripheral location and infiltrative margin predict invasive features of papillary thyroid microcarcinoma
}

\author{
Woo Kyung Lee,2,*, Jandee Lee ${ }^{3, *}$, Hyunji Kim³, Seul Gi Lee,3, Sun Hyung Choi ${ }^{3}$, Seonhyang Jeong2 \\ Hyeong Ju Kwon ${ }^{4}$, Sang Geun Jung ${ }^{5}$ and Young Suk Jo ${ }^{1,2}$
}

'Brain Korea 21 PLUS Project for Medical Science, Yonsei University, Seoul, Korea, Departments of ${ }^{2}$ Internal Medicine, ${ }^{3}$ Surgery, Open NBI Convergence Technology Research Laboratory, Yonsei University College of Medicine, Seoul, Korea, ${ }^{4}$ Department of Pathology, Yonsei University, Wonju College of Medicine, Wonju, Korea, and

${ }^{5}$ Department of Gynecological Oncology, Bundang CHA Medical Center, CHA University, Gyeonggi-do, Korea

Correspondence should be addressed to Y S Jo

Email

joys@yuhs.ac

\begin{abstract}
Objective: Tumor location in papillary thyroid microcarcinoma (PTMC) might determine tumor outgrowth from the thyroid gland. However, the clinical implications of tumor location and minimal extrathyroid extension (mETE) have not been well elucidated. We aimed to investigate the relationship between tumor location and mETE to predict the aggressiveness of PTMC.

Methods: A total of 858 patients with PTMC were grouped according to tumor location on ultrasonography: central ( $P$ PTMC) and peripheral PTMC (PPTMC). PTMC without mETE (PTMC-mETE(-)) was divided further according to margin shape: encapsulated (E-) or infiltrative (I-). To understand the molecular biologic characteristics of PTMC presenting with an I-margin and mETE, transcriptome data from TCGA-THCA were analyzed using Gene Set Enrichment Analysis (GSEA).

Results: pPTMC ( $n=807,94.1 \%)$ accounted for the majority of cases; mETE was identified only in pPTMC (403/807; 49.9\%). pPTMC-mETE(+) showed aggressive clinical characteristics that increased the odds ratio (OR) for lymph node metastasis (LNM). Interestingly, subgroup analysis of PTMC-mETE(-) revealed that the I-margin also increased the OR for LNM, independent of other clinical factors. GSEA of TCGA-THCA data suggested coordinated upregulation of genes related to epithelial-mesenchymal transition (EMT) in PTC with mETE. Immunohistochemical staining for laminin subunit gamma 2 (LAMC2), CD59, E-cadherin and vimentin showed that these markers of EMT were associated with progressive changes in E-margin PTMC-mETE(-), I-margin PTMC-mETE(-) and pPTMC-mETE(+).

Conclusion: mETE related to peripheral location of PTMC is an important predictor of tumor invasiveness, as is the I-margin, which presents with EMT features similar to mETE. I-margin PTMC-mETE(-) and PPTMC-mETE(+) might reflect the pattern of invasive PTMC.
\end{abstract}

\section{Introduction}

The incidence of papillary thyroid cancer (PTC), the most common endocrine malignancy, is increasing rapidly worldwide (1). This increase is due mainly to better detection of small PTC tumors, such as papillary thyroid microcarcinomas (PTMCs), which are defined as primary tumors measuring $1.0 \mathrm{~cm}$ or less (2). In general, PTMCs show low rates of loco-regional recurrence, distant metastases (DM) and disease-specific mortality $(3,4)$. However, some 
PTMCs exhibit aggressive features, including regional lymph node metastasis (LNM), DM and even cancerrelated death $(5,6)$. Because PTMC is defined by size criteria alone, not by other clinicopathological aggressive features, PTMCs may exhibit heterogeneity in terms of biological characteristics, resulting in controversy regarding clinical significance and management of the disease (7).

Extrathyroid extension (ETE) of the primary tumor was first reported as a poor prognostic factor in 1961 (8). Since then, ETE has been widely accepted as an important factor for predicting a poor prognosis and for determining surgical extent in patients with PTC $(9,10)$. The concept of minimal ETE (mETE) was introduced into the sixth edition of the TNM staging system (American Joint Committee on Cancer (AJCC)) in 2002; mETE is defined as an extension of the tumor to the thyroid capsule, sternothyroid muscle, or perithyroidal soft tissue. This is classified as stage T3. Therefore, patients with PTMC with mETE are categorized as having stage III disease (11). However, mETE has been removed from the T3 stage classification in the recently released 8th AJCC TNM staging system, which provides more accurate prediction of disease-specific survival (DSS) (12). Although the TNM staging system has discarded mETE $(13,14)$, the impact of mETE on PTC prognosis remains unclear. Some studies report that mETE is linked closely to a poor prognosis for patients with PTC $(15,16)$, but others report that the presence of mETE and/or gross ETE (gETE) has no significant effect on disease-free survival (DFS) $(17,18)$. In the case of PTMC, we believe that the situation is more complicated because tumor growth needs to reach the peripheral margin of the thyroid gland to be classed as mETE; however, the small size of a tumor is a confounding factor that limits the ability of tumor cells to reach the periphery of the thyroid gland.

Here, we examined the impact of tumor location on mETE and other clinicopathological features in PTMC. We also performed subgroup analysis according to the shape of the tumor margin to predict aggressiveness. Based on Gene Set Enrichment Analysis (GSEA) of data from TCGATHCA, we investigated the relationship between margin infiltration and mETE-related genes.

\section{Subjects and methods}

\section{Study population and subgroup classification}

The medical records of 858 patients who underwent thyroid surgery for PTMC (tumor size $\leq 1 \mathrm{~cm}$ ) at Severance Hospital, Yonsei College of Medicine (Seoul, South Korea) between January 2015 and May 2016 were reviewed retrospectively. All patients underwent preoperative ultrasonography (US) imaging and were suspected (preoperatively) of having thyroid cancer after US-guided fine-needle aspiration cytology. All US examinations were performed according to a standardized guideline by two radiologists specializing in thyroid imaging. PTMC associated with other malignancies or discovered incidentally after thyroidectomy was excluded. In addition, PTMC with gETE was excluded due to the small number of cases $(n=2)$. The study was approved by the Institutional Review Board at Severance Hospital. The requirements for patient approval and informed consent were waived due to the retrospective nature of the study.

The extent of thyroidectomy was based on prognostic factors. A hemithyroidectomy was performed if the following criteria were met on preoperative evaluation: a single lesion, no definite gETE, no clinical LNM and no personal history of radiation therapy to the head or neck. A total thyroidectomy was performed if there were multiple or bilateral lesions and if definite gETE or clinical LNM was discovered. Preoperative evaluation of cervical LN at levels (II-VI) was achieved by staging US and neck computed tomography. A prophylactic ipsilateral central compartment node dissection (CCND; pretracheal, prelaryngeal and paraesophageal) was performed in all cases for accurate staging of PTMC and to manage subclinical LNM. A bilateral CCND was performed if there were multiple lesions or bilateral lesions and if any sign of definite clinical LNM was discovered on preoperative imaging or during surgery.

To examine the impact of preoperative tumor location on mETE, the study population was divided into two groups, central (cPTMC) and peripheral PTMC (pPTMC), according to tumor location on axial views of US images. To group patients according to tumor location on US (axial view), the center of the tumor was set as the point at which two fixed major axes met. Next, the shortest distance from the center to the borderline of the thyroid gland (observed in the same plane) was measured. If the shortest distance was $\geq 0.5 \mathrm{~cm}$, the tumor was defined as cPTMC (Supplementary Fig. 1A, see section on supplementary data given at the end of this article); if not, the tumor was defined as pPTMC (Supplementary Fig. 1B). In the case of multifocal or bilateral tumors, the tumor location was recorded as that of the outermost tumor in the axial plane. The volume of each thyroid lobe $(\mathrm{mL})$ was calculated using the following equation: lobe volume $=\pi / 6 \times$ width $\times$ thickness $\times$ length. Total thyroid volume was estimated by summing the volumes calculated for the right and left thyroid lobes. To better understand 
biological diversity of PTMC with respect to tumor location and the shape of the tumor margin, we classified PTMC into five groups: encapsulated (E-) cPTMC (Group I), infiltrative (I-) cPTMC (Group II), E-pPTMC without mETE (Group III (E-pPTMC-mETE(-))), I-pPTMC without mETE (Group IV (I-pPTMC-mETE(-))) and pPTMC with mETE (Group V (pPTMC-ETE $(+))$ ). For this classification, we defined the I-margin as microscopic transcapsular tumor invasion observed by two independent pathologists.

\section{Hematoxylin and eosin staining and immunohistochemical (IHC) staining}

Formalin-fixed paraffin-embedded tissue sections $(4 \mathrm{~mm}$ thick) were stained with hematoxylin and eosin (H\&E). Tissue sections were deparaffinized in Histo-Clear II (HS202; National Diagnostics), rehydrated through a graded series of ethanol solutions, and then stained sequentially with hematoxylin (HHS16; Sigma) and eosin (HT110132; Sigma). Immunohistochemistry (IHC) was conducted using paraffin-embedded tissue sections (IHC-P). Briefly, sections were stained with antibodies specific for CD59 (sc-133170; Santa Cruz Biotechnology), LAMC2, (Laminin $\gamma-2$, sc-28330; Santa Cruz Biotechnology), CDH1 (AMAB90863; Sigma-Aldrich) or Vimentin (\#5741S; Cell Signaling Technology). For double staining, the VECTASTAIN ABC-AP Staining KIT AK-5000 (alkaline phosphatase, Standard; Vector Labs), the VECTOR Blue Alkaline Phosphatase (Blue AP) Substrate Kit SK-5300 (Vector Labs) and a DAB developing kit (REAL EnVision Detection System K5007; Agilent Technologies) were used according to the manufacturer's protocols. Stained slides were scanned and analyzed by an Aperio Scanscope AT Turbo instrument and Imagescope software (Aperio Technologies, Vista, CA, USA). For all analyses, 20 randomly chosen fields from each slide section were evaluated. Staining scores were determined as follows: negative, no staining or a faint/barely perceptible staining in any population of tumor cells; focal staining, either non-uniform or weak staining in at least $10 \%$ of tumor cells or an intense complete staining in $30 \%$ or less of tumor cells or diffuse staining, a uniform intense staining in more than $30 \%$ of the tumor cells.

\section{TCGA-THCA data analysis}

A public microarray database matched to clinicopathological data from The Cancer Genome Atlas (TCGA-THCA, https://cancergenome.nih.gov/; $n=505$ ) was scanned to identify the biological characteristics of PTC or PTMC presenting with mETE. For GSEA (http://software.broadinstitute.org/gsea/index.jsp) and analysis of clinicopathologic data, the data from TCGA-THCA were divided into three groups according to the presence of $\mathrm{mETE}$ or $\operatorname{gETE}: \operatorname{mETE}(-), \operatorname{mETE}(+)$ and gETE(+). GSEA predicted coordinated upregulation or downregulation of pre-organized signature gene sets according to the presence of mETE. Briefly, GSEA calculates an enrichment score (ES), which reflects the degree to which a gene set is over-represented at the top or bottom of a ranked list of genes. GSEA normalizes the ES to account for differences in gene set size and for differences in correlations between the gene sets and the expression dataset; thus, the normalized ES (NES) can be used to compare results across gene sets. Next, the GSEA calculates the false discovery rate (FDR) $q$-value, which is an estimated probability that a gene set with a given NES represents a false-positive finding. Because a FDR $q$-value of $25 \%$ indicates that the result is likely to be valid three out of four times, GSEA highlights enriched gene sets with a FDR $q$-value of $<25 \%$. It also estimates the statistical significance of the NES for a single gene set by calculating a nominal $P$ value. Here, the GSEA was performed using Hallmark gene sets version 6.1 according to the presence or absence of mETE. In this setting, a negative NES indicates gene set enrichment at the top of the ranked list for PTC-mETE(+). Among the enriched gene sets in a group, those with a nominal $P$ value $<0.05$ and a FDR $q$-value $<0.25$ were considered statistically significant. PTMC data $(n=22)$ from TCGATHCA were analyzed in the same way.

\section{Statistical analysis}

Continuous data were compared using a two-tailed Student's $t$ test or one-way ANOVA, and categorical data were compared using the chi-squared test or Fisher's exact test. Multivariable logistic regression models were used to investigate the impact of tumor location or mETE on the presence of LNM or tumor recurrence. The clinicopathological factors used to adjust for each model are described in the footnote to the relevant table. DFS in the $\operatorname{mETE}(-)$ and $\operatorname{mETE}(+)$ groups in TCGA-THCA was analyzed using a Kaplan-Meier estimator. All data are expressed as the mean \pm standard deviation (s.D.). Statistical analyses were performed using SPSS software (version 23; IBM Corp.) or GraphPad Prism 6 (GraphPad Software Inc.). $P<0.05$ was considered statistically significant. 


\section{Results}

\section{Peripheral location as a predictor of mETE in PTMC}

The clinicopathologic characteristics (according to tumor location on preoperative US) of the 858 patients with PTMC are listed in Table 1. Surprisingly, most PTMCs were located peripherally on the axial view (pPTMC, $n=807$, $94.1 \%)$. One reason for this may be the small size of the thyroid gland in Korean people. To verify these data, we measured the thyroid volume of all study patients; indeed, the result showed that the mean thyroid volume of the study patients was lower than that previous reported for Western populations (Supplementary Table 1) $(19,20,21)$. Analysis of clinicopathological factors according to PTMC location revealed that $403 / 807$ pPTMC cases presented with mETE; no mETE was observed in CPTMC cases $(P<0.0001)$. In addition, pPTMC showed more frequent pathological LNM $(P=0.035)$. However, there were no differences in other important factors such as tumor size, DM, and the BRAFV600E mutation between the groups (Table 1). Because we could find positive relationship between peripheral location and LNM, we conducted multivariable regression analysis to identify factors that increase the risk of LNM (Supplementary Table 2). Male sex, tumor size and mETE increased the odds ratio (OR) for LNM in our study cohort, while peripheral location did not. Based on these data, we postulated that peripheral location did not increase the risk of LNM directly; however, it may affect the frequency of LNM by increasing mETE.

\section{I-margin as a predictor of LNM in PTMC}

Even though no cPTMC cases presented with mETE, 10/51 cPTMC cases had LNM. When reviewing these ten cases, we found that all ten had an infiltrative tumor margin. Based on this observation, we classified the PTMC cases according to the histological shape of the tumor margin: encapsulated (E-margin) and infiltrative (I-margin) (Fig. 1A). Finally, combining these data with the presence or absence of mETE and tumor location, we divided PTMC cases into five groups: Group I, E-cPTMC $(n=7)$; Group II, I-cPTMC ( $n=44)$; Group III, E-pPTMC-mETE $(-)(n=77)$; Group IV, I-pPTMC-mETE(-) $(n=327)$; and Group V, pPTMC-ETE $(+)(n=403)$ (Table 2$)$.

Comparing the clinicopathological characteristics among the five groups revealed that LNM was observed most frequently in Group V; no LNM was detected in Group I, although the number of patients was small $(n=7)$ (Fig. 1B and Table 2). Furthermore, the incidence of LNM was markedly higher in Group IV than in

Table 1 Clinicopathological characteristics of PTMC according to tumor location. Data are expressed as the mean \pm S.D. or as number (\%).

\begin{tabular}{l} 
Characteristics \\
\hline$n(\%)$ \\
Shortest distance (cm) \\
Age (years) \\
Gender (female, \%) \\
Tumor size (cm) \\
Minimal ETE \\
Negative \\
Positive \\
Multifocality \\
Negative \\
Positive \\
Bilaterality \\
Negative \\
Positive \\
Pathological LNM \\
N0 \\
N1a/N1b \\
Distant metastasis \\
Negative \\
Positive \\
BRAFV600E mutation
\end{tabular}

\begin{tabular}{c}
\hline Central \\
$51(5.9)$ \\
$0.55 \pm 0.09$ \\
$45.5 \pm 11.8$ \\
$41(80.4)$ \\
$0.58 \pm 0.21$ \\
$51(100)$ \\
0 \\
$47(92.2)$ \\
$4(7.8)$ \\
$49(96.1)$ \\
$2(3.9)$ \\
$41(80.4)$ \\
$8(15.7) / 2(3.9)$ \\
51 \\
0 \\
$29(56.9)$
\end{tabular}

\begin{tabular}{|c|c|}
\hline Peripheral & P value* \\
\hline $807(94.1)$ & \\
\hline $0.30 \pm 0.10$ & $<0.0001$ \\
\hline $45.6 \pm 10.7$ & 0.966 \\
\hline $655(81.2)$ & 0.891 \\
\hline \multirow{2}{*}{$0.60 \pm 0.20$} & 0.462 \\
\hline & $<0.0001$ \\
\hline \multirow{2}{*}{\multicolumn{2}{|c|}{$\begin{array}{l}404(50.1) \\
403(49.9)\end{array}$}} \\
\hline & \\
\hline & $<0.0001$ \\
\hline \multicolumn{2}{|l|}{$557(69)$} \\
\hline \multicolumn{2}{|l|}{$250(31.0)$} \\
\hline & 0.030 \\
\hline \multicolumn{2}{|l|}{$687(85.1)$} \\
\hline \multicolumn{2}{|l|}{$120(14.9)$} \\
\hline & 0.035 \\
\hline \multicolumn{2}{|l|}{$524(64.9)$} \\
\hline \multicolumn{2}{|l|}{$243(30.1) / 40(5.0)$} \\
\hline & 1.000 \\
\hline \multicolumn{2}{|l|}{806 (99.9) } \\
\hline \multicolumn{2}{|l|}{$1(0.1)$} \\
\hline $498(61.7)$ & 0.490 \\
\hline
\end{tabular}

*Continuous variables were analyzed using a two-tailed Student's $t$ test, and categorical variables were analyzed using a two-tailed chi-squared test or Fisher's exact test.

ETE, extrathyroidal extension; LNM, lymph node metastasis; PTMC, papillary thyroid microcarcinoma. 
A
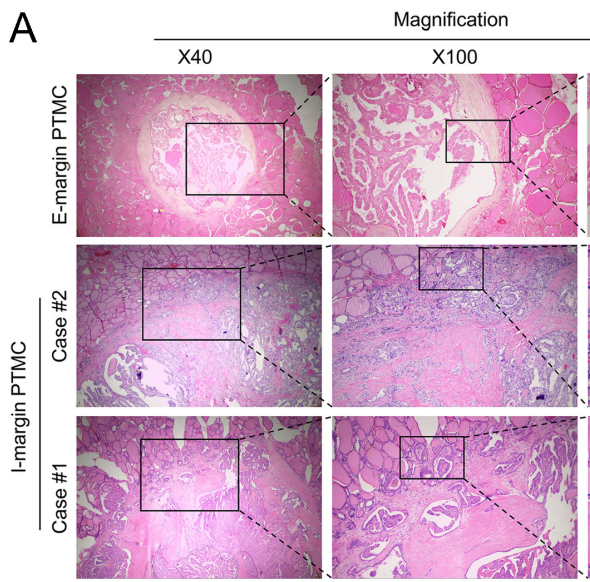

B

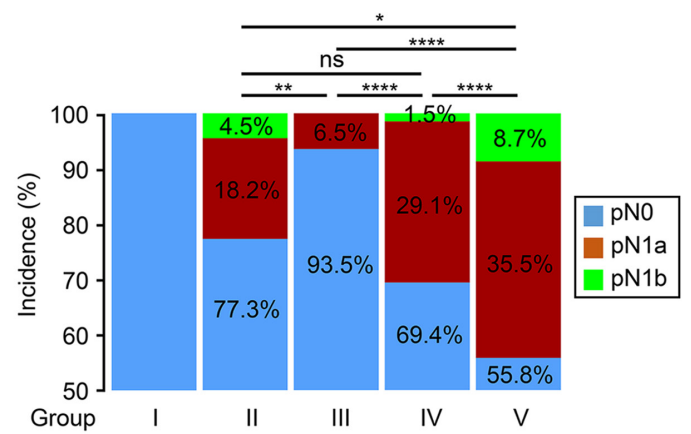

\section{Figure 1}

Clinical implications of the I-margin and minimal ETE in PTMC.

(A) Representative hematoxylin and eosin (H\&E) images of PTMCs with an E- and I-margin. (B) Comparison of LNM

incidence according to the classification of PTMC (i.e., METE, tumor location and margin shape; see manuscript text for details). Scale bars, $25 \mu \mathrm{m}$. ns, non-significant, $* P<0.05$, $\star * P<0.01, * * * P<0.001$ and $* * * * P<0.0001$. ETE, extrathyroidal extension; E-margin, encapsulated margin; I-margin, infiltrative margin; LNM, lymph node metastasis; PTMC, papillary thyroid microcarcinoma.

Group III $(P<0.0001)$. When comparing Group II with Group IV, we found no difference in LNM frequency $(P=0.284)$. However, LNM was more common in Group II than in Group III $(P=0.009)$. To determine the roles of I-margin and mETE, we further analyzed $P$ values obtained from statistical comparisons between two sets: I-margin versus E-margin and I-margin versus mETE (Group II/IV versus Group I/III and Group II/IV versus Group V, respectively). According to this analyses, poor clinical features, including multifocality, bilaterality and pathological LNM, were more common in patients with I-margin (Group II/IV) than in patients with E-margin (Group I/III). In addition, patients with mETE (Group V) showed more aggressive tumor behaviors, including tumor size, multifocality, bilaterality and pathological LNM, as well as BRAFV600E mutation, than patients with I-margin (Group II/IV) (Table 2).

Taken together, these findings suggested that I-margin and mETE might be predictors of aggressive tumor behaviors such as LNM. This was supported by multivariable regression analysis, which indicated clearly that I-margin and mETE increased the OR for LNM, even after adjusting for other important clinicopathological parameters (Table 3).

\section{TCGA-THCA analysis reveals that METE is a predictor of tumor aggressiveness and that it is closely related to EMT}

Although mETE of PTMC, a known risk factor for LNM, requires a peripherally located tumor, the risk of LNM was increased by the presence of an I-margin. Based on this observation, we decided to investigate the molecular biological changes generated by mETE to see whether they also occurred in PTMC with an I-margin. Using RNAseq data from TCGA-THCA, we first analyzed the clinicopathological characteristics of PTC according to the presence/absence of mETE or gETE. As expected, the largest tumors were identified in PTC-gETE $(+)$, and LNM was observed more frequently in PTC-mETE(+) or gETE(+) than in PTC-ETE(-). Furthermore, DFS was significantly lower for PTC-mETE(+) than for PTC-ETE(-) (Supplementary Fig. 2). After adjusting for other prognostic factors, multivariable regression analysis indicated that mETE increased the ORs for LNM and tumor recurrence (Supplementary Tables 3 and 4). Taken together, the data suggest that mETE in PTC is an indicator of poor prognosis. The data are supported by GSEA of TCGATHCA data, which identified a number of gene sets that show coordinately increased expression in PTC-mETE $(+)$ compared with PTC-ETE(-) (Fig. 2A); these include genes associated with epithelial-mesenchymal transition (EMT), inflammatory responses, angiogenesis and tumor necrosis factor-alpha (TNF $\alpha$ ) signaling via nuclear factor-kappa light chain enhancer of activated B cells (NF-кB). Interestingly, these gene sets were also expressed at higher levels in PTC with LNM (PTC-LNM(+)) than in PTC without LNM (PTC-LNM(-); Fig. 2B). Even though the number was too small to reach a definitive conclusion, analysis of PTMC $(n=22)$ cases from TCGA-THCA revealed the same trends with respect to mETE-related aggressive biological and clinicopathological features (Supplementary Fig. 3). 
Table 2 Clinicopathological characteristics according to tumor location, I-margin and minimal ETE in PTMC. Data are expressed as mean \pm S.D. or $n(\%)$.

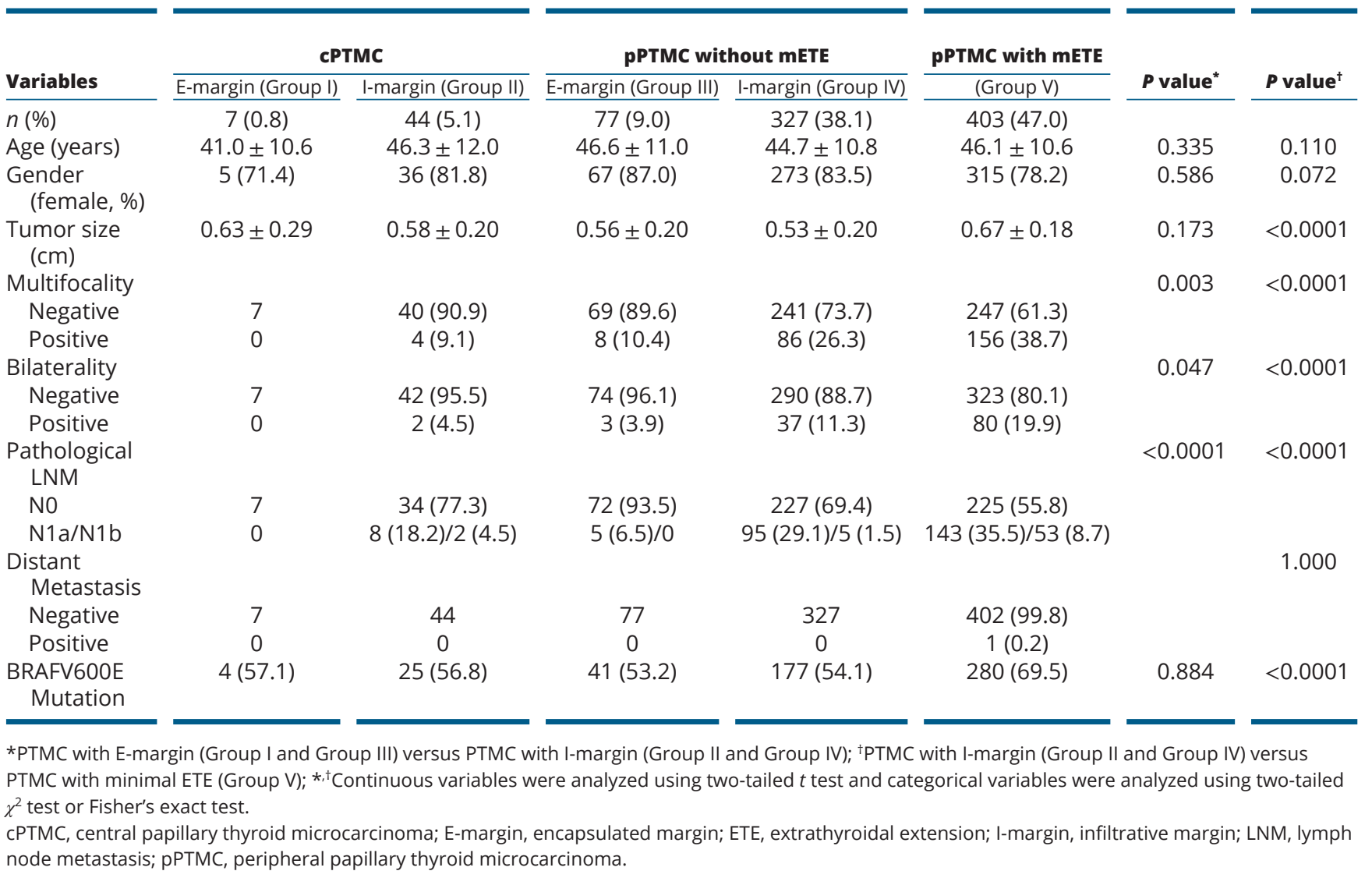

\section{EMT as a common feature of METE and I-margin}

Because we observed consistent upregulation of gene sets related to EMT in PTC-mETE(+) and PTC-LNM(+), we carefully reviewed individual genes listed in the EMT gene set. Among the 199 EMT-related genes, we decided to focus

Table 3 Multivariable regression analysis of margin infiltration $(n=371)$ and minimal ETE $(n=403)$ in PTMC for predicting LNM.

\begin{tabular}{|c|c|c|}
\hline Models* & LNM, OR $(95 \% \mathrm{CI})$ & P value \\
\hline Margin infiltration & $6.659(2.625-16.892)$ & $<0.0001$ \\
\hline Minimal ETE & $12.500(4.957-31.521)$ & $<0.0001$ \\
\hline Margin infiltrationt & $6.531(2.565-16.629)$ & $<0.0001$ \\
\hline Minimal ETE† & $12.470(4.924-31.580)$ & $<0.0001$ \\
\hline Margin infiltration $\ddagger$ & $6.300(2.460-16.130)$ & $<0.0001$ \\
\hline Minimal ETE $\ddagger$ & 9.988 (3.911-25.507) & $<0.0001$ \\
\hline
\end{tabular}

*Encapsulation ( $n=84$ ) is a reference category. tAdjusted for age and gender. $¥$ In addition to adjustment $¥$, adjusted for tumor size, multifocality, bilaterality and BRAFV600E mutation status. $\mathrm{Cl}$, confidence interval; ETE, extrathyroidal extension; LNM, lymph node metastasis; I-margin, infiltrative margin; PTMC, papillary thyroid microcarcinoma. on laminin subunit gamma 2 (LAMC2) and CD59; this is because both genes were highly enriched in PTC with mETE and PTMC with mETE (Fig. 2C and Supplementary Fig. 4A). In addition, these genes were expressed at higher levels in PTC than in normal thyroid tissues (Supplementary Fig. 4B). Similar to results obtained when using mETE as a variable, LNM in PTC cases with high expression of LAMC2 or CD59 was more common than in PTC with low expression of LAMC2 or CD59 (Fig. 2D). Based on the data from TCGATHCA, we further investigated expression of LAMC2 and CD59 in our five-group study cohort using IHC-P. Double IHC-P using antibodies specific for E-cadherin (Cadherin 1, CDH1) and vimentin (VIM) (representative markers of EMT) revealed that expression of $\mathrm{CDH} 1$ by tumor cells from Groups II to V was markedly lower than that by tumor cells from Group I (Fig. 3); there was no apparent difference in expression of VIM between groups. Interestingly, we observed clear expression of LAMC2 in Group V, but not in Groups I and III. Focal staining was observed in Groups II and IV (indicated by red arrows in Fig. 3). However, although staining for CD59 was strong in Group V, there 

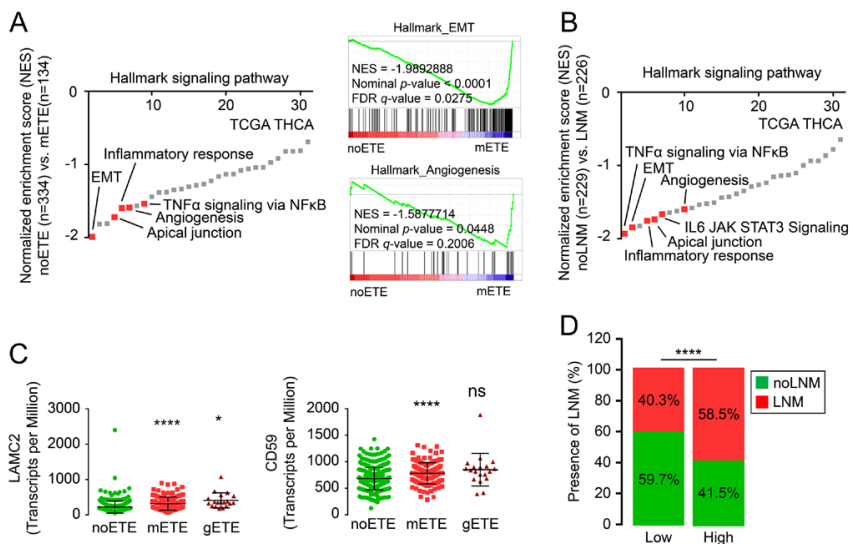

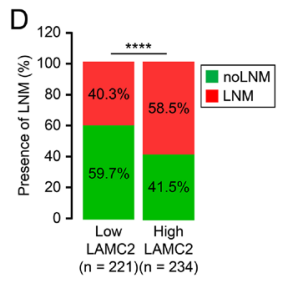

were no clear differences between staining intensity or pattern in Groups II, III, IV, and I (Fig. 3). Supporting our analyses of the role of I-margin and mETE on clinical features (Table 2), the expression of EMT-related genes, including CDH1, LAMC2 and CD 59, showed statistically significant difference across groups of patients (Table 4). Taken together, the data suggest that Group I showed slight changes in EMT gene expression (i.e., VIM), Groups II to IV showed some progression (indicated by disappearance of CDH1), Groups II and IV showed even more progression (focal expression of LAMC2), and finally, Group V showed extensive changes in expression of EMT-related genes (diffuse expression of LAMC2 and CD59) (Table 4).

\section{Discussion}

To the best of our knowledge, this is the first study to report that tumor location (on preoperative US) predicts mETE in

\section{Figure 2}

LAMC2 and CD59 as EMT markers in PTC with ETE or LNM. (A and B) The results of GSEA of data from TCGA-THCA indicate coordinated upregulation of certain gene sets in PTC with mETE (A) and LNM (B). Important hallmark signaling pathways are highlighted in red (nominal $P$ value $<0.05$; FDR $q$-value $<0.25$ ). (C) LAMC2 and CD59 expression according to ETE status. (D) LNM frequency according to LAMC2 and CD59 expression status. ns, non-significant, $* P<0.05$, and $\star * \star * P<0.0001$. CD59, CD59 molecule; EMT, epithelial-mesenchymal transition; gETE, gross ETE; GSEA, Gene Set Enrichment Analysis; LAMC2, laminin subunit gamma 2; mETE, minimal ETE; TCGA, The Cancer Genome Atlas; THCA, thyroid cancer.

PTMC. We also investigated the biological characteristics of mETE by analyzing public repository data from TCGATHCA. Interestingly, most PTMC cases (94.1\%) in our study population were located peripherally, which might be due to the fact that Asian populations have a smaller thyroid gland than Western populations $(19,20,21)$. In addition, we found that a peripheral location was an important determinant of mETE in PTMC. However, peripheral location was not an independent predictor of LNM, although PTMC-mETE(+) showed more aggressive features than PTMC-mETE(-), supported by multivariable binary regression analysis identified mETE as a powerful predictor of LNM. This suggests that factors other than location also affect tumor aggressiveness. Furthermore, GSEA of data from TCGA-THCA clearly indicated that mETE was associated with coordinated upregulation of gene sets related to EMT, tumor inflammation and angiogenesis (even in PTMC), thereby contributing to tumor invasion and recurrence. In line with our analysis

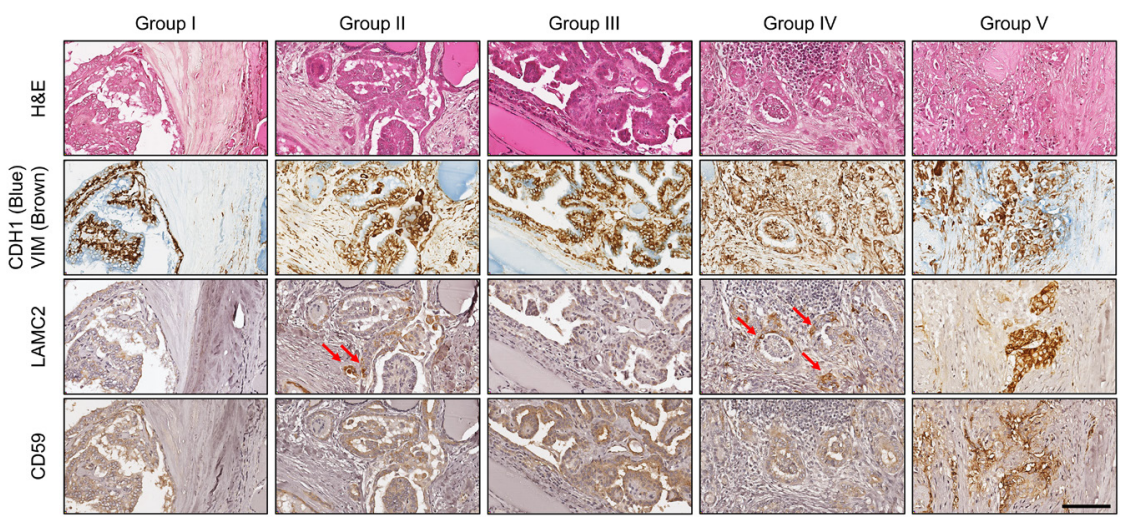

\section{Figure 3}

Representative IHC-P images showing expression of CDH1, VIM, LAMC2 and CD59 according to the classification system used herein. Images were obtained from the margin of tumors in each group. Scale bars, $100 \mu \mathrm{m}$. CDH1, cadherin 1, type 1, E-cadherin (epithelial); IHC-P, immunohistochemistry-paraffin; VIM, vimentin. 
Table 4 IHC-P results the five PTMC groups.

\begin{tabular}{|c|c|c|c|c|c|c|c|}
\hline \multirow[b]{2}{*}{ Variables } & \multicolumn{2}{|c|}{ сPTMC } & \multicolumn{2}{|c|}{ pPTMC without mETE } & \multirow[t]{2}{*}{ pPTMC with mETE } & \multirow[b]{2}{*}{ P value* } & \multirow[b]{2}{*}{ P value $^{\dagger}$} \\
\hline & $\begin{array}{l}\text { E-margin } \\
\text { (Group I) }\end{array}$ & $\begin{array}{l}\text { I-margin } \\
\text { (Group II) }\end{array}$ & $\begin{array}{l}\text { E-margin } \\
\text { (Group III) }\end{array}$ & $\begin{array}{c}\text { I-margin } \\
\text { (Group IV) }\end{array}$ & & & \\
\hline$n(\%)$ & $7(0.8)$ & $44(5.1)$ & $77(9.0)$ & 327 (38.1) & 403 (47.0) & & \\
\hline CDH1, diffuse & $6(86 \%)$ & 0 & 0 & 0 & 0 & $<0.0001$ & \\
\hline VIM, diffuse & $7(100 \%)$ & $44(100 \%)$ & 77 (100\%) & $327(100 \%)$ & $403(100 \%)$ & & \\
\hline \multicolumn{8}{|l|}{ LAMC2 } \\
\hline Focal & 0 & $11(25 \%)$ & $3(4 \%)$ & $140(43 \%)$ & 0 & $<0.0001$ & $<0.0001$ \\
\hline Diffuse & 0 & 0 & 0 & 0 & $302(75 \%)$ & & $<0.0001$ \\
\hline CD59, diffuse & 0 & 0 & 0 & 0 & $217(54 \%)$ & & $<0.0001$ \\
\hline
\end{tabular}

of TCGA-THCA, IHC-P confirmed changes in expression of EMT-related proteins with respect to location, margin shape and mETE.

The role of tumor location as a clinical parameter for predicting tumor aggressiveness is an important issue for Asian patients with PTMC $(22,23,24,25)$. Previous studies demonstrate that PTMC located in the upper pole, but not in inferior or lateral locations, is an important predictor of lateral $\operatorname{LNM}(22,23,24,25)$. However, we postulated that peripheral location of PTMC (not only in the upper pole) enables tumor cells to reach the margin of the thyroid gland, thereby facilitating extrathyroid invasion. Thus, we classified tumor location in terms of shortest distance from the tumor center to the thyroid margin (observed in the axial plane) to better reflect whether a tumor is peripherally located. Moreover, unlike previous studies, we found that, although peripheral tumor location was an important determinant of METE in PTMC, it is not an independent predictor of LNM; this is because other clinicopathological factors such as mETE and tumor size also affect LNM. Taken together, these observational data indicate that peripheral location might provide cancer cells with an opportunity to access the outer wall of the thyroid gland, although other biological changes are required for LNM.

Many previous reports show that PTMC is more aggressive in Korean patients than in Western patients (26). These aggressive features have been explained by the high incidence of BRAFV600E-positive PTMC in Korea $(27,28,29,30)$. However, although the BRAFV600E mutation is the most useful diagnostic and prognostic molecular marker for predicting aggressiveness of thyroid cancer, including ETE and LNM (31), its prognostic impact remains controversial $(32,33)$. Here, we found no difference in the prevalence of BRAFV600E mutation in those with CPTMC or PPTMC, indicating a non-significant effect of that driver mutation in either group. However, the subgroup pPTMC-mETE(+) showed a high frequency of the BRAFV600E mutation, suggesting that mETE might be a malignant characteristic of aggressive thyroid cancer. To put this another way, the location of PTMC might be random, but mETE might result from a malignant biological process triggered by interactions between tumor cells and the microenvironment. We found it interesting that an I-margin, a histopathological feature of invasion, was observed in a significant proportion of cPTMC cases $(86.3 \%)$. Although there was no mETE in cPTMC (according to our criteria for defining the location of the tumor), I-cPTMC showed aggressive tendencies, such as LNM. However, the aggressive behavior was not as marked as that of pPTMC with mETE, suggesting that tumor location is an important determinant for mETE and provides an opportunity for progression to a more aggressive phenotype. Compatible with this idea, although PTMC location was itself not an independent predictor for LNM, mETE related to tumor location was the most statistically powerful determinant of LNM. In summary, peripheral tumor location might allow cancer cells to acquire aggressive characteristics that result in mETE and LNM. Conversely, if an aggressive tumor arises in the periphery, it would be expected to spread beyond the gland more quickly and easily.

The clinico-biological significance of mETE in thyroid cancer remains unclear, although gETE is an important predictive factor for determining surgical extent in patients with PTMC because of its contribution to the high incidence of local recurrence and locoregional invasion (34). Recent studies report that mETE 
has no impact on DFS of patients with PTC, although gETE reduces DFS $(35,36,37,38)$. By contrast, some studies suggest that mETE increases the risk of PTC acquiring aggressive characteristics and the risk of tumor recurrence $(16,39,40)$. To investigate whether mETE is a possible answer to this controversy, we performed GSEA according to the presence of mETE or LNM using data from TCGA-THCA. The data showed that mETE was related to coordinate upregulation of genes involved in EMT, tumor inflammation and angiogenesis. Along with these biological changes, multivariable logistic regression analysis of data from TCGA-THCA clearly showed that not only gETE, but also mETE, was an independent predictor for LNM and tumor recurrence. Therefore, we should not overlook biological changes in the tumor related to mETE when we decide on the treatment strategy for patients with mETE at an early stage. In addition, we need to consider the implications of the EMT-related changes seen in Groups II and IV.

This retrospective observational study has some limitations. First, patients were enrolled from a single tertiary medical center, which may result in selection bias. Second, the number of patients with central tumor location was relatively small. To verify the power of statistical calculation influenced by sample size, we performed post hoc power calculation using PASS software (version 12, NCSS, LLC, Kaysville, UT, USA). Between cPTMC and pPTMC, group sample sizes of 51 in cPTMC and 807 in pPTMC achieved a power of $76 \%$ at a 0.05 significance level. Between E-margin and I-margin, group sample sizes of 84 in E-margin and 371 in I-margin achieved 100\% power at a 0.05 significance level for detecting a difference between group proportions of 0.2360 . These results of intergroup comparisons indicated that the sample sizes in our study were sufficient for practical application. Nevertheless, the number of cPTMCs was smaller than that of pPTMCs, and further study of a larger volume of data is needed. Third, we could not evaluate the impact of mETE on DFS due to the short follow-up period. In fact, we first tried to investigate the effect of tumor location on PTMC biology. Thus, to define tumor location exactly, we needed to enroll PTMC patients for whom high-resolution US images were available. Also, it was important that all US examinations were performed by skilled radiologists who specialized in thyroid imaging according to the latest standardized guidelines. In addition, we discussed our proposed classification of PTMC with these specialists. Therefore, we enrolled PTMC patients that had visited the hospital recently and thus had relatively short follow-up periods.
To overcome this limitation, we analyzed DFS of patients according to the presence of mETE when analyzing data from TCGA-THCA (PTC including PTMC, $n=458$ ) and when performing subgroup analysis of PTMC cases $(n=22)$. However, these multi-center cohorts might be heterogenous. Also, our analysis of DFS of PTMC cases was still limited by the small number of cases $(4 / 22)$ showing recurrence.

Another concern of our study design for tumor location was that we arbitrary divided PTMC into two groups (central vs. peripheral tumor) based on $0.5 \mathrm{~cm}$, because the maximum radius of PTMC cannot exceed $0.5 \mathrm{~cm}$. By using this definition, cPTMC cannot reach the thyroid border before becoming more than $1 \mathrm{~cm}$ in size. We believe that we were actually able to obtain welldefined groups because the mean tumor size and shortest distance of cPTMC were $0.58 \pm 0.21 \mathrm{~cm}$ and $0.55 \pm 0.09 \mathrm{~cm}$, respectively, indicating that the distance between the border of cPTMC and the thyroid borderline was 2 $3 \mathrm{~mm}(0.26 \pm 0.1 \mathrm{~cm})$, which is considered a potentially safe distance in active surveillance of PTMC. In addition, based on our definition, the distance from the thyroid borderline was significantly different between cPTMC (Group I/II) and pPTMC (Group III/IV). However, there was no statistically significant difference in the distance to thyroid border between PTMC with I-margin versus PTMC with E-margin (Group II/IV and Group I/III). In fact, the frequency of several factors, such as mETE, varies depending on distance. When the lower value of the distance to thyroid border was set to cut-off value, the clinical significance of distinguishing central PTMC was declined. For example, when we changed our definition for central PTMC from $\geq 5 \mathrm{~mm}$ to $\geq 3 \mathrm{~mm}$, we observed the presence of mETE in 195 cases (44.8\%) out of total 435 central PTMC cases.

With respect to the defining tumor location, we did not use a $3 \mathrm{D}$ classification because we believed that the axial view provides information about tumor location within the thyroid gland in relation to the surrounding structures. For instance, most PTMCs located at the upper and lower poles in this study were located in peripheral regions (99.4 and 96.8\%, respectively). This means that, because the upper or lower pole portion comprises a smaller volume of the thyroid gland than other portions, the distance from the tumor center to the thyroid borderline might be shorter and that the tumor can be relatively closer to the thyroid borderline. We propose that our definition reflects previous reports suggesting that PTMC located in the upper or lower pole generally presents poor prognosis $(24,25,41)$. 
In summary, mETE related to peripheral location of PTMC is an important predictor of tumor invasiveness. The I-margin is another predictor of invasiveness of PTMC. Therefore, I-margin PTMC-mETE(-) and pPTMC$\mathrm{mETE}(+)$ might reflect patterns of invasive PTMC.

\section{Supplementary data}

This is linked to the online version of the paper at https://doi.org/10.1530/ EJE-18-1025

\section{Declaration of interest}

The authors declare that there is no conflict of interest that could be perceived as prejudicing the impartiality of this study.

\section{Funding}

Y S J was supported by National Research Foundation of Korea (NRF) grants funded by the Korean government (MEST) (NRF-2018R1A2B6004179) and by a grant from the Korea Health Technology R\&D Project through the Korea Health Industry Development Institute, funded by the Ministry of Health \& Welfare, Republic of Korea (HI15C2334). J L was supported by a National Research Foundation of Korea (NRF) grant funded by the Korea government (MEST) (NRF-2017R1D1A1A09000916) and by a faculty research grant from Yonsei University College of Medicine (6-2017-0073).

\section{Acknowledgement}

The authors thank Ji Young Kim, Hoyoung Kim and Hwanju Lee for the technical support.

\section{References}

1 Sosa JA \& Udelsman R. Total thyroidectomy for differentiated thyroid cancer. Journal of Surgical Oncology 200694 701-707. (https:// doi.org/10.1002/jso.20695)

2 Hughes DT, Haymart MR, Miller BS, Gauger PG \& Doherty GM. The most commonly occurring papillary thyroid cancer in the United States is now a microcarcinoma in a patient older than 45 years. Thyroid 201121 231-236. (https://doi.org/10.1089/thy.2010.0137)

3 Pazaitou-Panayiotou K, Capezzone M \& Pacini F. Clinical features and therapeutic implication of papillary thyroid microcarcinoma. Thyroid 200717 1085-1092. (https://doi.org/10.1089/thy.2007.0005)

4 Pacini F. Thyroid microcarcinoma. Best Practice and Research: Clinical Endocrinology and Metabolism 201226 381-389. (https://doi. org/10.1016/j.beem.2011.10.006)

5 Pellegriti G, Scollo C, Lumera G, Regalbuto C, Vigneri R \& Belfiore A. Clinical behavior and outcome of papillary thyroid cancers smaller than $1.5 \mathrm{~cm}$ in diameter: study of 299 cases. Journal of Clinical Endocrinology and Metabolism 200489 3713-3720. (https://doi. org/10.1210/jc.2003-031982)

6 Malandrino P, Pellegriti G, Attard M, Violi MA, Giordano C, Sciacca L, Regalbuto C, Squatrito S \& Vigneri R. Papillary thyroid microcarcinomas: a comparative study of the characteristics and risk factors at presentation in two cancer registries. Journal of Clinical Endocrinology and Metabolism 201398 1427-1434. (https://doi. org/10.1210/jc.2012-3728)

7 Ito Y, Miyauchi A \& Oda H. Low-risk papillary microcarcinoma of the thyroid: a review of active surveillance trials. European Journal of Surgical Oncology 201844 307-315. (https://doi.org/10.1016/j. ejso.2017.03.004)
8 Woolner LB, Beahrs OH, Black BM, McCONAHEY WM \& Keating FR. Classification and prognosis of thyroid carcinoma. A study of 885 cases observed in a thirty year period. American Journal of Surgery 1961102 354-387. (https://doi.org/10.1016/0002-9610(61)90527-X)

9 Sugitani I, Kasai N, Fujimoto Y \& Yanagisawa A. A novel classification system for patients with PTC: addition of the new variables of large $(3 \mathrm{~cm}$ or greater) nodal metastases and reclassification during the follow-up period. Surgery 2004135 139-148. (https://doi. org/10.1016/S0039)

10 Verburg FA, Mader U, Tanase K, Thies ED, Diessl S, Buck AK, Luster M $\&$ Reiners C. Life expectancy is reduced in differentiated thyroid cancer patients $>/=45$ years old with extensive local tumor invasion, lateral lymph node, or distant metastases at diagnosis and normal in all other DTC patients. Journal of Clinical Endocrinology and Metabolism 201398 172-180. (https://doi.org/10.1210/jc.2012-2458)

11 Edge SB, Compton CC. The American Joint Committee on Cancer: the 7th edition of the AJCC cancer staging manual and the future of TNM. Annals of Surgical Oncology 201017 1471-1474. (https://doi. org/10.1245/s10434-010-0985-4)

12 Tuttle RM, Haugen B \& Perrier ND. Updated American Joint Committee on Cancer/tumor-node-metastasis staging system for differentiated and anaplastic thyroid cancer (eighth edition): what changed and why? Thyroid 201727 751-756. (https://doi. org/10.1089/thy.2017.0102)

13 Kim TH, Kim YN, Kim HI, Park SY, Choe JH, Kim JH, Kim JS, Oh YL, Hahn SY, Shin JH et al. Prognostic value of the eighth edition AJCC TNM classification for differentiated thyroid carcinoma. Oral Oncology 201771 81-86. (https://doi.org/10.1016/j. oraloncology.2017.06.004)

14 Haugen BR. 2015 American Thyroid Association Management Guidelines for adult patients with thyroid nodules and differentiated thyroid cancer: what is new and what has changed? Cancer $2017 \mathbf{1 2 3}$ 372-381. (https://doi.org/10.1002/cncr.30360)

15 Shin JH, Ha TK, Park HK, Ahn MS, Kim KH, Bae KB, Kim TH, Choi CS, Kim TK, Bae SK et al. Implication of minimal extrathyroidal extension as a prognostic factor in papillary thyroid carcinoma. International Journal of Surgery 201311 944-947. (https://doi. org/10.1016/j.ijsu.2013.06.015)

16 Yin DT, Yu K, Lu RQ, Li X, Xu J \& Lei M. Prognostic impact of minimal extrathyroidal extension in papillary thyroid carcinoma. Medicine 201695 e5794. (https://doi.org/10.1097/ MD.0000000000005794)

17 Hu A, Clark J, Payne RJ, Eski S, Walfish PG \& Freeman JL. Extrathyroidal extension in well-differentiated thyroid cancer: macroscopic vs microscopic as a predictor of outcome. Archives of Otolaryngology: Head and Neck Surgery 2007133 644-649. (https://doi. org/10.1001/archotol.133.7.644)

18 Arora N, Turbendian HK, Scognamiglio T, Wagner PL, Goldsmith SJ, Zarnegar R \& Fahey TJ 3rd. Extrathyroidal extension is not all equal: implications of macroscopic versus microscopic extent in papillary thyroid carcinoma. Surgery 2008144 942-947; discussion 947-948. (https://doi.org/10.1016/j.surg.2008.07.023)

19 Sari R, Balci MK, Altunbas H \& Karayalcin U. The effect of body weight and weight loss on thyroid volume and function in obese women. Clinical Endocrinology 200359 258-262. (https://doi. org/10.1046/j.1365-2265.2003.01836.x)

20 Hegedus L, Perrild H, Poulsen LR, Andersen JR, Holm B, Schnohr P, Jensen $\mathrm{G} \&$ H Hansen JM. The determination of thyroid volume by ultrasound and its relationship to body weight, age, and sex in normal subjects. Journal of Clinical Endocrinology and Metabolism 1983 56 260-263. (https://doi.org/10.1210/jcem-56-2-260)

21 Oberhofer R, Ober A, Oberkofler F \& Amor H. Thyroid gland volumes of healthy adults in an area with endemic goiter. Acta Medica Austriaca 198916 38-41.

22 Ito Y, Tomoda C, Uruno T, Takamura Y, Miya A, Kobayashi K, Matsuzuka F, Kuma K \& Miyauchi A. Papillary microcarcinoma of the 
thyroid: how should it be treated? World Journal of Surgery 200428 1115-1121. (https://doi.org/10.1007/s00268-004-7644-5)

23 Kwak JY, Kim EK, Youk JH, Kim MJ, Son EJ, Choi SH \& Oh KK. Extrathyroid extension of well-differentiated papillary thyroid microcarcinoma on US. Thyroid 200818 609-614. (https://doi. org/10.1089/thy.2007.0345)

24 Kwak JY, Kim EK, Kim MJ, Son EJ, Chung WY, Park CS \& Nam KH. Papillary microcarcinoma of the thyroid: predicting factors of lateral neck node metastasis. Annals of Surgical Oncology 200916 1348-1355. (https://doi.org/10.1245/s10434-009-0384-x)

25 Lee DJ, Lee KH, Kim JH, Kwon KH, Yoon DY \& Rho YS. Posterosuperior lesion has a high risk of lateral and central nodal metastasis in solitary papillary thyroid cancer. World Journal of Surgery 201539 387-392. (https://doi.org/10.1007/s00268-014-2839-x)

26 Lee J, Rhee Y, Lee S, Ahn CW, Cha BS, Kim KR, Lee HC, Kim SI, Park CS \& Lim SK. Frequent, aggressive behaviors of thyroid microcarcinomas in Korean patients. Endocrine Journal 200653 627-632. (https://doi.org/10.1507/endocrj.K06-013)

27 Jo YS, Li S, Song JH, Kwon KH, Lee JC, Rha SY, Lee HJ, Sul JY, Kweon GR, Ro HK et al. Influence of the BRAF V600E mutation on expression of vascular endothelial growth factor in papillary thyroid cancer. Journal of Clinical Endocrinology and Metabolism 200691 3667-3670. (https://doi.org/10.1210/jc.2005-2836)

28 Chung KW, Yang SK, Lee GK, Kim EY, Kwon S, Lee SH, Park DJ, Lee HS, Cho BY, Lee ES et al. Detection of BRAFV600E mutation on fine needle aspiration specimens of thyroid nodule refines cyto-pathology diagnosis, especially in BRAF600E mutationprevalent area. Clinical Endocrinology 200665 660-666. (https://doi. org/10.1111/j.1365-2265.2006.02646.x)

29 Jo YS, Huang S, Kim YJ, Lee IS, Kim SS, Kim JR, Oh T, Moon Y, An S, Ro HK et al. Diagnostic value of Pyrosequencing for the BRAF V600E mutation in ultrasound-guided fine-needle aspiration biopsy samples of thyroid incidentalomas. Clinical Endocrinology 200970 139-144. (https://doi.org/10.1111/j.1365-2265.2008.03293.x)

30 Joo JY, Park JY, Yoon YH, Choi B, Kim JM, Jo YS, Shong M \& Koo BS. Prediction of occult central lymph node metastasis in papillary thyroid carcinoma by preoperative BRAF analysis using fine-needle aspiration biopsy: a prospective study. Journal of Clinical Endocrinology and Metabolism 201297 3996-4003. (https://doi.org/10.1210/ jc. 2012-2444)

31 Nikiforova MN \& Nikiforov YE. Molecular diagnostics and predictors in thyroid cancer. Thyroid 200919 1351-1361. (https://doi. org/10.1089/thy.2009.0240)

32 Ito Y, Yoshida H, Maruo R, Morita S, Takano T, Hirokawa M, Yabuta T, Fukushima M, Inoue $\mathrm{H}$, Tomoda $\mathrm{C}$ et al. BRAF mutation in papillary thyroid carcinoma in a Japanese population: its lack of correlation with high-risk clinicopathological features and disease-free survival of patients. Endocrine Journal 200956 89-97. (https://doi. org/10.1507/endocrj.K08E-208)

33 Lee KC, Li C, Schneider EB, Wang Y, Somervell H, Krafft M, Umbricht CB \& Zeiger MA. Is BRAF mutation associated with lymph node metastasis in patients with papillary thyroid cancer? Surgery 2012152 977-983. (https://doi.org/10.1016/j.surg.2012.08.019)

34 Ortiz S, Rodriguez JM, Soria T, Perez-Flores D, Pinero A, Moreno J \& Parrilla P. Extrathyroid spread in papillary carcinoma of the thyroid: clinicopathological and prognostic study. Otolaryngology: Head and Neck Surgery 2001124 261-265. (https://doi.org/10.1067/ mhn.2001.113141)

35 Asanuma K, Kusama R, Maruyama M, Fujimori M \& Amano J. Macroscopic extranodal invasion is a risk factor for tumor recurrence in papillary thyroid cancer. Cancer Letters 2001164 85-89. (https:// doi.org/10.1016/S0304-3835(00)00698-4)

36 Ito Y, Tomoda C, Uruno T, Takamura Y, Miya A, Kobayashi K, Matsuzuka F, Kuma K \& Miyauchi A. Prognostic significance of extrathyroid extension of papillary thyroid carcinoma: massive but not minimal extension affects the relapse-free survival. World Journal of Surgery 200630 780-786. (https://doi.org/10.1007/s00268-005-0270-z)

37 Ito Y, Tomoda C, Uruno T, Takamura Y, Miya A, Kobayashi K, Matsuzuka F, Kuma K \& Miyauchi A. Minimal extrathyroid extension does not affect the relapse-free survival of patients with papillary thyroid carcinoma measuring $4 \mathrm{~cm}$ or less over the age of 45 years. Surgery Today 200636 12-18. (https://doi.org/10.1007/s00595-0053090-8)

38 Fukushima M, Ito Y, Hirokawa M, Miya A, Shimizu K \& Miyauchi A. Prognostic impact of extrathyroid extension and clinical lymph node metastasis in papillary thyroid carcinoma depend on carcinoma size. World Journal of Surgery 201034 3007-3014. (https://doi.org/10.1007/ s00268-010-0776-x)

39 Jung SP, Kim M, Choe JH, Kim JS, Nam SJ \& Kim JH. Clinical implication of cancer adhesion in papillary thyroid carcinoma: clinicopathologic characteristics and prognosis analyzed with degree of extrathyroidal extension. World Journal of Surgery 201337 1606-1613. (https://doi.org/10.1007/s00268-013-2034-5)

40 Park JS, Chang JW, Liu L, Jung SN \& Koo BS. Clinical implications of microscopic extrathyroidal extension in patients with papillary thyroid carcinoma. Oral Oncology 201772 183-187. (https://doi. org/10.1016/j.oraloncology.2017.02.008)

41 Brito JP, Ito Y, Miyauchi A \& Tuttle RM. A clinical framework to facilitate risk stratification when considering an active surveillance alternative to immediate biopsy and surgery in papillary microcarcinoma. Thyroid 201626 144-149. (https://doi.org/10.1089/ thy.2015.0178)

Received 25 December 2018

Revised version received 28 April 2019

Accepted 29 May 2019 